\title{
Prevalência de ansiedade em estudantes de enfermagem de uma instituição de ensino superior
}

\author{
Prevalence of anxiety in nursing students at a higher education institution \\ Prevalencia de ansiedad en estudiantes de enfermería de una institución de educación superior
}

Recebido: 30/01/2022 | Revisado: 04/02/2022 | Aceito: 11/02/2022 | Publicado: 17/02/2022

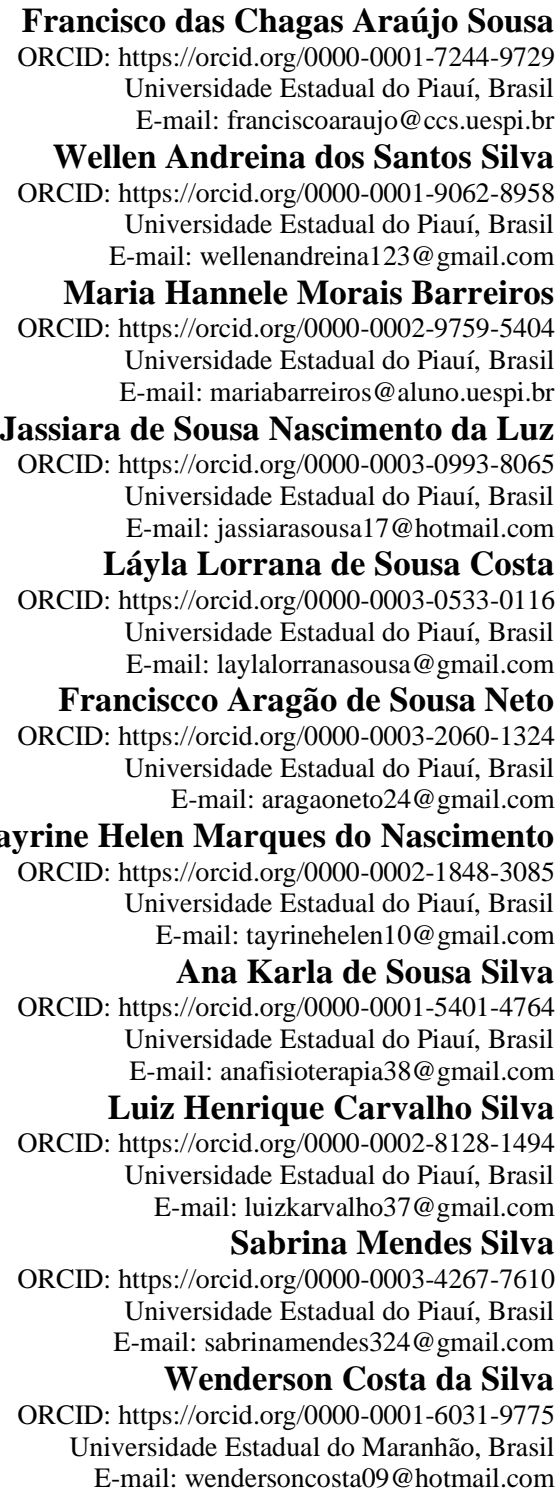

\section{Resumo}

A ansiedade é um sentimento negativo e está relacionada a situações que os indivíduos vivenciam no seu cotidiano. Quando essa ansiedade traz preocupação excessiva e malefícios na vida dos indivíduos, ela torna-se uma patologia. Este estudo teve como objetivo determinar a prevalência de ansiedade em estudantes de enfermagem de uma instituição de ensino superior. Trata-se de um estudo descritivo, transversal, com abordagem quantitativa. Os participantes da pesquisa foram os alunos regularmente matriculados no curso de Enfermagem de uma instituição pública de ensino superior distribuídos em 10 turmas. A amostra da pesquisa foi composta por 50 alunos. Quantos aos resultados, quatro (8\%) estudantes eram do sexo masculino e $46(92 \%)$ do sexo feminino, onde a faixa etária predominante foi 21-30 anos, e mais da metade dos acadêmicos praticam atividades físicas ocasionalmente. Em relação a quantidade e qualidade do sono, notou-se que mais da metade (56\%) dorme apenas 6h/dia, e 54\% 
classificam o sono como insatisfatório, ou seja, incapaz de fornecer um descanso físico e mental adequado. Verificouse que $86 \%$ dos acadêmicos, consideram-se pessoas ansiosas, entretanto apenas $22 \%$ afirmaram utilizar tratamento medicamentoso para ansiedade. Ao avaliar a presença dos diferentes níveis de ansiedade, através do Inventário de Beck, constatou-se que a maioria apresentou Ansiedade Grave (32\%) e Ansiedade Leve (30\%). Conclui-se que a universidade e os professores, devem procurar maneiras de diminuir essa carga de exigências e pressões sobre os alunos, tornando a experiência acadêmica leve e positiva sem comprometer o aprendizado e as responsabilidades necessárias para formação do profissional.

Palavras-chave: Transtornos de ansiedade; Estudantes de enfermagem; Universidades; Estilo de vida saudável.

\begin{abstract}
Anxiety is a negative feeling and is related to situations that individuals experience in their daily lives. When this anxiety brings excessive worry and harm to individuals' lives, it becomes a pathology. This study aimed to determine the prevalence of anxiety in nursing students from a higher education institution. This is a descriptive, cross-sectional study with a quantitative approach. The research participants were students regularly enrolled in the Nursing course of a public higher education institution, distributed in 10 classes. The research sample consisted of 50 students. As for the results, four (8\%) students were male and $46(92 \%)$ were female, where the predominant age group was 21-30 years old, and more than half of the students practice physical activities occasionally. Regarding the quantity and quality of sleep, it was noted that more than half (56\%) sleep only $6 \mathrm{~h} /$ day, and $54 \%$ classify sleep as unsatisfactory, that is, unable to provide adequate physical and mental rest. It was found that $86 \%$ of students consider themselves to be anxious people, however only $22 \%$ reported using drug treatment for anxiety. When evaluating the presence of different levels of anxiety, through the Beck Inventory, it was found that the majority had Severe Anxiety (32\%) and Mild Anxiety (30\%). It is concluded that the university and the professors, should look for ways to reduce this load of demands and pressures on students, making the academic experience light and positive without compromising the learning and responsibilities necessary for professional training.
\end{abstract}

Keywords: Anxiety disorders; Students, nursing; Universities; Healthy lifestyle.

\title{
Resumen
}

La ansiedad es un sentimiento negativo y está relacionado con situaciones que las personas experimentan en su vida diaria. Cuando esta ansiedad trae una preocupación excesiva y un daño a la vida de las personas, se convierte en una patología. Este estudio tuvo como objetivo determinar la prevalencia de ansiedad en estudiantes de enfermería de una institución de educación superior. Se trata de un estudio descriptivo, transversal con enfoque cuantitativo. Los participantes de la investigación fueron estudiantes matriculados habitualmente en el curso de Enfermería de una institución pública de educación superior, distribuidos en 10 clases. La muestra de investigación estuvo constituida por 50 estudiantes. En cuanto a los resultados, cuatro (8\%) estudiantes eran varones y $46(92 \%)$ mujeres, donde el grupo de edad predominante fue de 21-30 años, y más de la mitad de los estudiantes practican actividades físicas de forma ocasional. En cuanto a la cantidad y calidad del sueño, se observó que más de la mitad (56\%) duerme solo 6h / día, y el 54\% clasifica el sueño como insatisfactorio, es decir, incapaz de proporcionar un adecuado descanso físico y mental. Se encontró que el $86 \%$ de los estudiantes se consideran personas ansiosas, sin embargo, solo el 22\% informó haber usado un tratamiento farmacológico para la ansiedad. Al evaluar la presencia de diferentes niveles de ansiedad, a través del Inventario de Beck, se encontró que la mayoría presentaban Ansiedad severa (32\%) y Ansiedad leve (30\%). Se concluye que la universidad y los profesores, deben buscar formas de reducir esta carga de demandas y presiones sobre los estudiantes, haciendo la experiencia académica liviana y positiva sin comprometer los aprendizajes y responsabilidades necesarios para la formación profesional.

Palabras clave: Trastornos de ansiedad; Estudiantes de enfermería; Universidades; Estilo de vida saludable.

\section{Introdução}

A ansiedade é um sentimento negativo e está relacionada a situações que os indivíduos vivenciam no seu cotidiano. Quando essa ansiedade traz preocupação excessiva e malefícios na vida dos indivíduos, ela torna-se uma patologia (De Melo et al., 2021).

Tal condição emocional é encarada como uma reação natural, um instinto de sobrevivência decorrido por uma sensação desagradável de apreensão, como uma resposta do organismo frente a algo que represente uma adversidade, ou que seja percebido dessa maneira pelo indivíduo. A ansiedade pode tornar-se crônica com manifestações como fobias, estados dissociativos, obsessões ou compulsões. Ressalta-se que as condições físicas e psíquicas de ansiedade, além de serem resultado de conflitos no inconsciente, são derivadas de hábitos, ou seja, de comportamentos adquiridos ao longo da vida (Pereira et al., 2019). 
Diante do contexto pandêmico atual, várias mudanças aconteceram no padrão de vida da população e, consequentemente no cotidiano dos acadêmicos. Cerca de metade dos discentes já enfrentam insegurança, medo, sensação de perda e mudanças no humor como resultado da pandemia, comparativamente aos períodos normais. Ademais, as inúmeras implicações como a ruptura da rotina de estudos, o adiamento das tarefas e atividades acadêmicas, o atraso do cronograma do curso, repercutem nos planos do futuro profissional e na busca pela independência e estabilidade financeira, baseada na expectativa da formação universitária. Essas implicações afetam diretamente o emocional do discente, potencializando a ansiedade (Gundim et al., 2021).

Quando comparados a universitários de outros cursos, os alunos de enfermagem possuem fatores adicionais que podem acarretar ansiedade como: a experiência da prática clínica, o relacionamento com o paciente, o receio de cometer erros, o sofrimento psíquico, somados às circunstâncias em que precisam lidar com a iminência de morte. Esses aspectos tendem a desencadear ansiedade no decorrer do curso, refletindo negativamente sobre o bem-estar, e se tornam um obstáculo para um bom desempenho durante e após a graduação (Fernandes et al., 2018).

Desse modo, é notório a importância de se estudar ansiedade em acadêmicos de enfermagem. Quanto mais abordado for o assunto, os primeiros sinais de ansiedade podem ser percebidos pelo próprio estudante e à medida que se expande o acesso à informação, consequentemente aumenta o conhecimento, possibilitando que o problema se torne menos difícil de ser solucionado se detectado no início (Da silva et al., 2021).

Diante do exposto o objetivo geral deste estudo foi determinar a prevalência de ansiedade em estudantes de enfermagem de uma instituição de ensino superior. E especificamente determinar o perfil sociodemográfico dos estudantes de enfermagem; identificar sinais de ansiedade entre os estudantes através do inventário de ansiedade de Beck; detectar possíveis graus de ansiedade entre os estudantes; e correlacionar níveis de ansiedade com fatores como o uso de medicamentos, consumo de bebidas alcoólicas e horas de sono.

\section{Metodologia}

Trata-se de um estudo descritivo, transversal, com abordagem quantitativa. A abordagem quantitativa será adequada à realização desse trabalho pelo fato de seu objeto de pesquisa ser um fenômeno mensurável e também por se tratar de um tema que exige objetividade dos seus resultados.

Este projeto de pesquisa foi aprovado pelo Comitê de Ética em Pesquisa da UNIP, CAAE: 29826720.4.0000.5512 e os acadêmicos que aceitaram participar da pesquisa assinaram um termo de consentimento livre e esclarecido (TCLE) eletrônico. A pesquisa obedeceu aos critérios éticos com base na Resolução no 466/2012 do Conselho Nacional de Saúde CNS. Portanto, a coleta de dados desse estudo somente será realizada após a aprovação do projeto pelo Comitê de Ética em Pesquisa.

Os participantes da pesquisa foram os alunos regularmente matriculados no curso de Enfermagem da referida UESPI distribuídos em 10 turmas. A amostra da pesquisa foi composta por 50 alunos.

Os dados da pesquisa foram coletados através de questionários eletrônicos contendo questões relativas ao tema do estudo, dados sociodemográficos e dados de saúde dos participantes. Para a identificação de sinais de ansiedade entre os estudantes foi utilizado o Beck Anxiety Inventory (BAI) - Inventário de Ansiedade de Beck. O inventário é autoaplicável e possui 21 perguntas, sendo considerado um bom instrumento psicométrico para deteç̧ão de ansiedade.

As perguntas do Inventário de Ansiedade de Beck são de múltipla escolha, sendo cada uma delas com um escore associado: Absolutamente não (0); Ligeiramente (1); Moderadamente (2) e Gravemente (3). A soma dos escores varia entre $0 \mathrm{e}$ 63 pontos; de acordo com as respostas os acadêmicos foram classificados na faixa de perfil ansioso, da seguinte forma: 0 a 7 (Mínimo); 8 a 15 (Suave); 16 a 25 (Moderado) e 26 a 63 (Grave). 
O questionário de saúde utilizado incluiu questões referentes a: diagnóstico de doença, utilização de medicamentos de uso contínuo e indutores ou depressores do sistema nervoso central, hábitos de vida, horas diárias de sono e qualidade do sono.

Os participantes do estudo foram abordados e esclarecidos quanto ao conteúdo e a finalidade da pesquisa, de forma virtual, através de um questionário eletrônico, sendo solicitados a ler atenciosamente o instrumento de coleta e responder as perguntas caso estivesse de acordo em participar.

Foram incluídos na pesquisa os alunos com idade mínima de 18 anos, devidamente matriculados no curso de enfermagem no período do momento da coleta dos dados e que estiverem frequentando as aulas, que aceitaram participar da pesquisa, assinando o termo de consentimento livre e esclarecido eletrônico (TCLE). Os critérios de exclusão foram: alunos menores de idade, que não estejam frequentando as aulas, mesmo estando matriculados, além daqueles que se recusarem a assinar o TCLE.

A análise dos resultados obtidos foi processada por intermédio de cálculos estatísticos, colocados em números absolutos e percentuais, da forma que melhor demonstrar a quantidade amostral e análise das respostas dos sujeitos.

\section{Resultados e Discussão}

A coleta de dados da instituição resultou em um total de 50 acadêmicos distribuídos em 10 turmas, sendo 4 (8\%) estudantes do sexo masculino e 46 (92\%) estudantes do sexo feminino, que estão devidamente matriculados no curso de Enfermagem em uma Instituição de Ensino Superior Pública. A faixa etária predominante foi 21-30 anos, e mais da metade dos acadêmicos praticam atividades físicas ocasionalmente, como descrito na Tabela 1.

Tabela 1. Distribuição dos dados sociodemográficos. Teresina, PI, Brasil, 2021.

\begin{tabular}{lcc}
\hline Variáveis & $\mathbf{N}$ & $\mathbf{\%}$ \\
\hline Sexo & 46 & 92,0 \\
Feminino & 4 & 8,0 \\
Masculino & & \\
Faixa etária & 19 & 38,0 \\
$18-20$ anos & 31 & 62,0 \\
$21-30$ anos & & 18,0 \\
Prática de atividade física & 9 & 58,0 \\
Não & 29 & 24,0 \\
Ocasionalmente & 12 & \\
Com frequência & & \\
\hline
\end{tabular}

Fonte: Sousa et al. (2021).

De acordo com a Tabela 2, em relação a quantidade e qualidade do sono que são um dos fatores predisponentes para o desenvolvimento da ansiedade, notou-se que mais da metade (56\%) dorme apenas 6h/dia, e 54\% classificam o sono como insatisfatório, ou seja, incapaz de fornecer um descanso físico e mental adequado.

Tabela 2. Distribuição da quantidade e qualidade do sono. Teresina, PI, Brasil, 2021.

\begin{tabular}{lcc}
\hline Variáveis & N & \% \\
\hline Tempo de sono & 9 & 18,0 \\
Menos de 6 horas & 28 & 56,0 \\
6 horas & 13 & 26,0 \\
7 horas ou mais & & \\
Qualidade do sono & 23 & 46,0 \\
Satisfatória & 27 & 54,0 \\
Não satisfatória & & \\
\hline
\end{tabular}

Fonte: Sousa et al. (2021). 
Além disso, o uso excessivo de aparelhos eletrônicos também consiste em um fator agravante da ansiedade, por conta do excesso de informação e por ser prejudicial ao sono, durante a pesquisa é perceptível que $80 \%$ dos participantes utilizam aparelhos eletrônicos por mais de 6h (Gráfico 1).

Gráfico 1. Distribuição do uso de aparelhos eletrônicos/dia. Teresina, PI, Brasil, 2021.

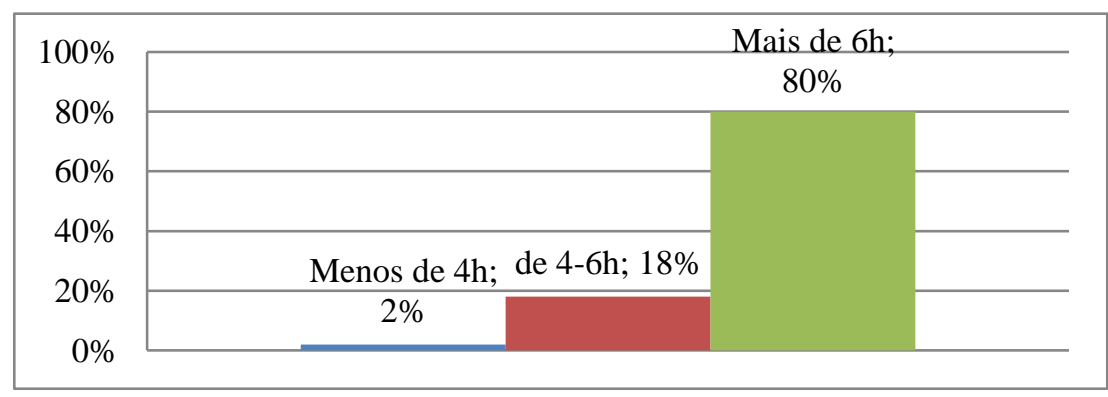

Fonte: Sousa et al. (2021).

Conforme a Tabela 3, verificou-se que $86 \%$ dos acadêmicos, consideram-se pessoas ansiosas, entretanto apenas $22 \%$ afirmaram utilizar tratamento medicamentoso para ansiedade.

Tabela 3. Distribuição da ansiedade e tratamento medicamentoso. Teresina, PI, Brasil, 2021.

\begin{tabular}{lcc}
\hline Variáveis & N & \% \\
\hline Se consideram ansiosos & 43 & 86,0 \\
Sim & 7 & 14,0 \\
Não & & 22,0 \\
Utilizam medicamentos para ansiedade & 11 & 78,0 \\
Sim & 39 & \\
Não
\end{tabular}

Fonte: Sousa et al. (2021).

Ao avaliar a presença dos diferentes níveis de ansiedade, através do Inventário de Beck, constatou-se que dos 50 alunos a maioria apresentam Ansiedade Grave (32\%) e Ansiedade Leve (30\%), como mostra a Tabela 4.

Tabela 4. Distribuição da frequência dos níveis de ansiedade. Teresina, PI, Brasil, 2021.

\begin{tabular}{lcc}
\hline Níveis de ansiedade & $\mathbf{N}$ & $\%$ \\
\hline Grau Mínimo & 8 & 16 \\
Ansiedade Leve & 15 & 30 \\
Ansiedade Moderada & 11 & 22 \\
Ansiedade Grave & 16 & 32 \\
\hline
\end{tabular}

Fonte: Sousa et al. (2021).

De acordo com os dados obtidos durante a pesquisa, é perceptível a correlação negativa estaticamente significativa entre a ansiedade e a vida acadêmica. Com os dados da tabela 4, nota-se que $84 \%$ dos estudantes da amostra total, apresentam ansiedade leve, moderada ou grave, sendo predominante na população de estudo a presença de ansiedade grave, entretanto apenas 22\% utilizam ou já utilizaram medicamentos para ansiedade. Portanto, através desses dados é possível constatar o quanto a vivência acadêmica exige grande responsabilidade de qualificação para ingresso no mercado de trabalho, como realização de trabalhos e atividades, preparação para as avaliações, além disso, dedicar tempo para produção científica, atividade essas que influenciam diretamente no desenvolvimento e/ou agravamento do quadro de ansiedade durante o ensino superior. 
Nesse contexto, foi enfatizado na pesquisa, alguns fatores predispostos para o aparecimento da ansiedade, como os hábitos de vida (prática de exercício físico), quantidade e qualidade de sono, e o tempo de utilização de aparelhos eletrônicos (Lúcio et al., 2019). A atividade física é benéfica de várias formas, pois promove a movimentação do corpo e relaxamento da mente (Lourenço et al., 2017). Em virtude desse tempo dedicado para a promoção do cuidado pessoal e bem-estar, retira o foco das obrigações da universidade. Apenas $24 \%$ dos estudantes relataram praticar exercício com frequência, é uma porcentagem relativamente negativa, pois exercita-se é uma forma de autocuidado, que previne contra várias doenças, inclusive transtornos de ansiedade.

O sono é imprescindível para a existência dos seres humanos, ele promove homeostasia entre o corpo e a mente, garantindo o descanso necessário para a realização de atividades diárias. O cotidiano dentro de uma universidade, é marcado por inúmeros afazeres e responsabilidades, uma vez que os discentes estão se preparando para entrada no mercado de trabalho, para essa formação e aprendizado é necessário muito estudo e qualificação (Dos Santos et al., 2020), o que requer uma quantidade relevante de tempo do acadêmico, principalmente os que participaram do estudo, pois o curso é de tempo integral (manhã/tarde), o que exige ainda mais tempo do aluno. Diante de várias atividades, torna-se difícil ainda conciliar a vida social e o autocuidado, o que acaba contribuindo para perturbação do sono desses estudantes. Conforme mostra a Tabela 3, a quantidade e qualidade de sono dos alunos estão prejudicadas, pois, a maioria referiu dormir apenas $6 \mathrm{~h} / \mathrm{dia}(56 \%)$ e $54 \%$ classificaram a qualidade do seu sono como não satisfatória, incapaz de restabelecer totalmente o bem-estar físico e psíquico necessário para realização das atividades diárias de forma efetiva.

Atualmente os aparelhos eletrônicos estão imersos em nosso cotidiano, facilitando a comunicação e disseminação de informação. Durante a graduação esses meios tecnológicos são constantemente utilizados, principalmente no contexto pandêmico atual, em que foi necessário o isolamento social, para conter a disseminação do coronavírus, com isso os estudantes precisaram ter aulas de forma remota, resultando no aumento do tempo voltado para uma tela de celular/computador (Mendes Leão et al., 2018). Como referido no Gráfico 1, em que $80 \%$ utilizam os aparelhos mais de $6 \mathrm{~h} / \mathrm{dia}$. No entanto, o uso excessivo desses aparelhos pode provocar efeitos maléficos a visão, a postura corporal, ao humor, gerar dependência, causar insônia, além de colaborar para a promoção de transtornos de ansiedade pelo excesso de informação fornecido e o aumento da vivência no meio virtual, provocando distanciamento entre as pessoas no mundo real (Nascimento et al., 2020).

Dessa forma, é evidente que ao ingressar em um ambiente do ensino superior, o acadêmico se depara com várias mudanças, como a forma de estudo, o aumento das cargas horárias, com grandes exigências e pressão (Fernandes et al., 2018), principalmente os discentes de Enfermagem que tem uma responsabilidade maior, pois vai trabalhar com as vidas de outras pessoas, por isso precisam ter uma qualificação e aprendizado adequado, para exercer essa função de forma eficiente e segura, isso acaba gerando uma elevada cobrança desses alunos, propiciando um desafio para conseguir administrar essa grande responsabilidade, com efetuar todas as atividades requeridas pelo curso, conciliar com a vida social, com o bem-estar emocional e uma rotina de sono regular saudável. Com isso, afeta a saúde mental e a qualidade de vida desses estudantes, gerando uma vulnerabilidade psicológica, o que pode acarretar outras doenças mentais, além da ansiedade.

\section{Conclusão}

Diante dos resultados expostos pela pesquisa, é perceptível como a vida acadêmica influencia no bem-estar físico e psíquico, assim nota-se a necessidade de medidas para promoção da saúde mental dos discentes do curso de Enfermagem, com o intuito de reduzir/evitar o aparecimento de sintomas referentes ao transtorno de ansiedade. Dessa forma, é imprescindível a implementação de apoio psicológico ao estudante durante a graduação, para que haja o acompanhamento e orientação sobre como conduzir uma vida psicologicamente saudável, dentro de uma instituição de ensino superior. Além disso, incluir a realização de atividades em grupos, com objetivo de promover a integração social gerando momentos de descontração e 
formação de vínculos entre os alunos, assim como promover rodas de conversas para discutir sobre os sintomas provocados pela ansiedade, com a intenção de detecção dos participantes que apresentam os indícios para ansiedade, e realizar encaminhamento ao serviço de psicologia para tratamento, evitando agravos.

Portanto, a universidade e os professores, devem procurar maneiras de diminuir essa carga de exigências e pressões sobre os alunos, tornando a experiência acadêmica leve e positiva sem comprometer o aprendizado e as responsabilidades necessárias para formação do profissional.

\section{Referências}

Silva, A. M. G., Silva, C. E. N., Silva, A. F., Oliveira, D. F. de, Sousa, T. J., \& Estrela, F. M. (2021). Ansiedade em acadêmicos de enfermagem e suas complicações futuras. Revista de Divulgação Científica Sena Aires, 10(2), 269-279. https://doi.org/10.3623/revisa.v\%.n\%.p713\%.

De Melo, H. E., Severian, P. F. G., Eid, L. P., Souza, M. R. de, Sequeira, C. A. C., Souza, M. da G. G., \& Pompeo, D. A. (2021). Impacto dos sintomas de ansiedade e depressão na autoeficácia percebida em estudantes de enfermagem. Acta Paulista de Enfermagem, 34, eAPE01113. https://doi.org/10.37689/actaape/2021ao01113.

Fernandes, M. A., Vieira, F. E. R., Silva, J. S. e, Avelino, F. V. S. D., \& Santos, J. D. M. (2018). Prevalence of anxious and depressive symptoms in college students of a public institution. Revista Brasileira de Enfermagem, 71(suppl 5), 2169-2175. https://doi.org/10.1590/0034-7167-2017-0752.

Gundim, V. A., Encarnação, J. P., Santos, F. C., Santos, J. E., Vasconcellos, E. A., \& Souza, R. C. de. (2021). Saúde mental de estudantes universitários durante a pandemia de covid-19. Rev. Baiana Enferm, 35, e37293-e37293. https://pesquisa.bvsalud.org/controlecancer/resource/pt/biblio1149683 ? src $=$ similardocs.

Mendes Leão, A., Gomes, I., José, M., Ferreira, M., Pamplona, L., \& Cavalcanti, G. (2018). Revista Brasileira De Educação Médica, 42 (4), 55-65. https://doi.org/10.1590/1981-52712015v42n4RB20180092.

Lourenço, B. S., Peres, M. A. A., Porto, I. S., Oliveira, R. M. P., \& Dutra, V. F. D. (2017). Escola Anna Nery, 21(3), e20160390. https://doi.org/10.1590/21779465-EAN-2016-0390.

Lúcio, S. S. R., Medeiros, L. G. S., Barros, D. R., Ferreira, O. D. L., \& Rivera, G. A. (2019). Temas em Saúde, 260-274. https://temasemsaude.com/wpcontent/uploads/2019/03/fippsi15.pdf.

Nascimento, D. S., Veloso, F. C. S., Sampaio, D. L., Oliveira, M. J. C., \& Kassar, S. B. (2020). Consequências do uso excessivo do smartphone na saúde mental de estudantes universitários: Uma revisão integrativa. Cadernos Brasileiros de Saúde Mental/Brazilian Journal of Mental Health, 12(32), 1-15. https://periodicos.ufsc.br/index.php/cbsm/article/view/69812.

Pereira, F. L. R., Medeiros, S. P., Fernandes Salgado, R. G., Castro, J. N. A. de, \& Oliveira, A. M. N. de. (2019). Anxiety signs experienced by nursing undergraduates / Manifestações de ansiedade vivenciadas por estudantes de enfermagem. Revista de Pesquisa: Cuidado é Fundamental Online, 11(4), 880886. https://doi.org/10.9789/2175-5361.2019.v11i4.880-886.

Dos Santos, A. F., Mussi, F. C., Pires, C. G. S., Santos, C. A. S. T., \& Paim, M. A. S. (2020). Qualidade do sono e fatores associados em universitários de enfermagem. Acta Paulista de Enfermagem, 33, eAPE20190144. https://doi.org/10.37689/acta-ape/2020ao0144. 